\title{
Knockdown expression and hepatic deficiency reveal an atheroprotective role for SR-BI in liver and peripheral tissues
}

\author{
Thierry Huby, ${ }^{1}$ Chantal Doucet, ${ }^{1}$ Christiane Dachet, ${ }^{1}$ Betty Ouzilleau, ${ }^{1}$ Yukihiko Ueda, ${ }^{2}$ \\ Veena Afzal, ${ }^{3}$ Edward Rubin,, ${ }^{3}$ M. John Chapman, ${ }^{1}$ and Philippe Lesnik'

\begin{abstract}
1INSERM U551, Université Pierre et Marie Curie-Paris 6, Dyslipoproteinemia and Atherosclerosis Research Unit, Hôpital de la Pitié, Paris, France. ${ }^{2}$ Horizontal Medical Research Organization, Graduate School of Medicine, Kyoto University, Shogoin, Sakyo-ku, Kyoto, Japan. ${ }^{3}$ Lawrence Berkeley National Laboratory, Genomics Division, Berkeley, California, USA.
\end{abstract}

\begin{abstract}
Scavenger receptor SR-BI has been implicated in HDL-dependent atheroprotective mechanisms. We report the generation of an SR-BI conditional knockout mouse model in which SR-BI gene targeting by loxP site insertion produced a hypomorphic allele (hypomSR-BI). Attenuated SR-BI expression in hypomSR-BI mice resulted in 2-fold elevation in plasma total cholesterol (TC) levels. Cre-mediated SR-BI gene inactivation of the hypomorphic SR-BI allele in hepatocytes (hypomSR-BI-KO liver) was associated with high plasma TC concentrations, increased plasma free cholesterol/TC (FC/TC) ratio, and a lipoprotein-cholesterol profile typical of $S R-\mathrm{BI}^{-/-}$mice. Plasma TC levels were increased 2-fold in hypomSR-BI and control mice fed an atherogenic diet, whereas hypomSR-BI-KO ${ }^{\text {liver }}$ and $S R-B I^{-/-}$mice developed severe hypercholesterolemia due to accumulation of FC-rich, VLDL-sized particles. Atherosclerosis in hypomSR-BI mice was enhanced (2.5-fold) compared with that in controls, but to a much lower degree than in hypomSR-BI-KO ${ }^{\text {liver }}$ (32-fold) and $S R-B I^{-/-}$(48-fold) mice. The latter models did not differ in either plasma lipid levels or in the capacity of VLDL-sized lipoproteins to induce macrophage cholesterol loading. However, reduced atherosclerosis in hypomSR-BI-KOliver mice was associated with decreased lesional macrophage content as compared with that in $S R-B I^{-/-}$mice. These data imply that, in addition to its major atheroprotective role in liver, SR-BI may exert an antiatherogenic role in extrahepatic tissues.
\end{abstract}

\section{Introduction}

Plasma concentrations of HDL cholesterol (HDL-C) are inversely proportional to the risk for premature development of atherosclerosis. One of the major atheroprotective actions of HDL particles involves the transport of excess cholesterol from peripheral tissues to the liver for excretion, a process known as reverse cholesterol transport (RCT). Understanding of the molecular events implicated in the formation and intravascular recycling of HDL particles, as well as the identification of key factors that control cholesterol flux through the plasma HDL pool, is critical to the development of innovative therapeutic strategies to enhance HDL-mediated RCT.

Scavenger receptor class B type 1, a physiologically relevant HDL receptor expressed in a variety of tissues, including vascular endothelial cells $(1,2)$, smooth muscle cells (3), and macrophages (4), mediates selective cellular uptake of HDL-associated cholesteryl esters (CEs) in the liver but also in adrenals (reviewed in ref. 5). Indeed, SR-BI is a major determinant of HDL metabolism in rodents. Hepatic overexpression of SR-BI in mice markedly reduces plasma HDL-C levels and increases biliary secretion of cholesterol (6-9). Conversely, partial to complete deficiency of this receptor results in an SR-BI dose-dependent elevation in plasma HDL-C with the appearance of large cholesterol-rich HDL particles $(10,11)$.

Nonstandard abbreviations used: CE, cholesteryl ester; FC, free cholesterol; HDL-C, HDL cholesterol; HFC, high-fat, high-cholesterol, and cholic acid-containing; PDZK1, PDZ domain containing 1-deficient; RCT, reverse cholesterol transport; SR-BI, scavenger receptor class B type 1; TC, total cholesterol; TG, triglyceride. Conflict of interest: The authors have declared that no conflict of interest exists. Citation for this article: J. Clin. Invest. 116:2767-2776 (2006). doi:10.1172/JCI26893.
In humans, naturally occurring mutations that affect SR-BI function and that clearly establish the relevance of this receptor to HDL-C levels and RCT have not been detected to date. However, the human homolog of SR-BI, Cla-1, exhibits tissue distribution similar to and selective HDL-CE uptake identical to those of murine SR-BI (reviewed in 5). Moreover, epidemiological studies have identified polymorphisms in the Cla-1 gene that are associated not only with plasma HDL-C or LDL cholesterol (LDL-C) levels, but also with lipoprotein particle size and coronary artery disease, suggesting that SR-BI may be intimately involved in HDL metabolism in humans (12-14).

A substantial body of evidence supports an atheroprotective role for SR-BI in mice. Thus, complete deficiency of SR-BI was associated with significantly increased atherosclerosis in mice fed a high-fat/cholesterol diet (15) as well as with accelerated atherosclerosis development in the atherosclerosis-prone apoE-KO mouse model (16). Conversely, hepatic overexpression of SR-BI led to reduction in murine atherosclerosis (6, $17,18)$. The antiatherogenic impact of SR-BI expressed in the liver most likely involves its role in the final step of RCT, with potential elevation in cholesterol flux to the liver (19). However, additional mechanisms may contribute to this effect, as SR-BI transgene-mediated reduction of atherosclerosis in the human apoB100 transgenic mouse model was primarily associated with reduction in plasma non-HDL cholesterol levels (17). It has been difficult to gain a clear understanding of the tissue-specific contribution of SR-BI to protection against atherogenesis because of the multifunctional properties attributed to this receptor and the wide spectrum of ligands to which SR-BI binds; these 
$\mathbf{A}$

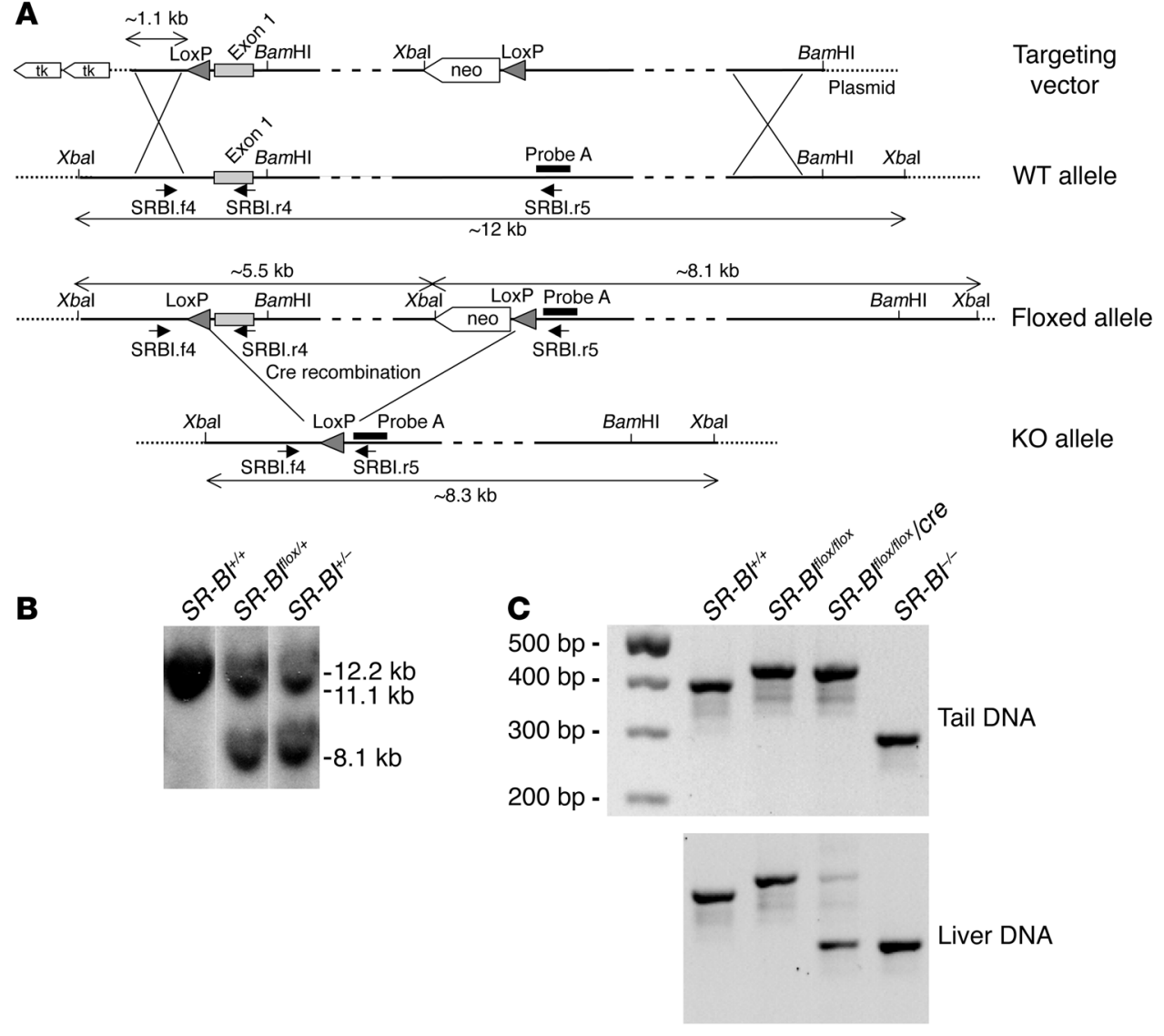

Figure 1

Targeting strategy and conditional deletion of the SR-BIflox allele. (A) Structural organization of the $5^{\prime}$ region of the wild-type, floxed, and knockout SR-BI allele. The 2 loxP sites flanking the SR-BI exon 1 are shown as arrowheads. The relative positions of probe A used for Southern blot analysis, primers (short arrows) used for PCR screening, and endonuclease sites for $\mathrm{BamHI}$ and $\mathrm{Xbal}$ are indicated. Xbal-restricted fragments for each allele are depicted by double-headed arrows. Cre-mediated deletion of the SR-BI ${ }^{\text {flox }}$ allele to generate the SR-BI-KO allele was achieved by transfection of the Cre recombinase cDNA in SR-BIflox/+ ES cells. $S R-B I^{+-}$ES cells were then used to generate SR-BI-KO mice. Mice with hepatic SR-BI deficiency were generated by interbreeding of $S R-B f^{f l o x} / f l o x$ mice with Alb-Cre mice, which express the Cre recombinase in hepatocytes. (B) Southern blot analysis of Xbal-digested ES cell genomic DNA using probe A. (C) PCR analysis of tail and liver genomic DNA using a mix of SRBI.f4, SRBI.r4, and SRBI.r5 primers. Primer pair SRBI.f4 and SRBI.r4 discriminates for the presence or absence of the 5'-loxP sequence upstream SR-BI exon 1. The sizes of the PCR products for the WT and SR-BI floxed alleles are $391 \mathrm{bp}$ and $427 \mathrm{bp}$, respectively. Primer pair SRBI.f4 and SRBI.r5 identifies the SR-BI-KO allele (288 bp).

include not only HDL and oxidized LDL, but also anionic phospholipids, apoptotic cells, and the acute-phase protein serum amyloid A, all of which are relevant to atherogenesis. In addition to its capacity to facilitate selective cellular HDL-CE uptake, other tissue-specific mechanisms underlying the antiatherogenic properties of SR-BI may include: (a) activation of eNOS, and thus production of antiatherogenic nitric oxide in the vascular endothelium $(2,20)$; (b) contribution to the first step of RCT by mediation of cellular free cholesterol (FC) efflux from peripheral tissues, including macrophages (21-23); (c) induction of apoptosis in damaged endothelial cells (24); (d) recognition and phagocytosis of apoptotic cells by macrophages (25); and (e) cellular uptake of lipoprotein-associated $\alpha$-tocopherol, thereby contributing to increased antioxidant bioavailability in cells of the arterial wall $(26,27)$.

\section{Results}

Earlier studies in PDZ domain containing 1-deficient (PDZK1deficient) mice have provided insight into the impact of tissuespecific ablation of the SR-BI isoform in the hepatocyte (95\%) and small intestine (50\%) on cholesterol homeostasis (28). In order to evaluate the relative contributions of hepatic versus extrahepatic SR-BI expression to cholesterol homeostasis and atherogenesis, we developed an SR-BI conditional knockout mouse model by Cre/loxp technology. However, lox $\mathrm{P}$ targeting of the $\mathrm{SR}-\mathrm{BI}$ gene resulted in reduced SR-BI expression in all tissues, generating a hypomorphic SR-BI mouse line in which SR-BI can be specifically deleted by Cre/lox $\mathrm{P}$ recombination. Our data reveal that while atherosclerosis was enhanced in mice with reduced expression of SR-BI (hypomSR-BI mice), aortic lesion formation was dramatically accelerated in mice with Cre-mediated deletion of hepatic SR-BI (hypomSR-BI$\mathrm{KO}^{\text {liver}}$ ) mice. Marked accumulation of atherogenic VLDL-sized lipoproteins enriched in FC most probably accounted for increased atherosclerosis in hypomSR-BI-KO liver mice. However, whereas hypomSR-BI$\mathrm{KO}^{\text {liver }}$ and $\mathrm{SR}-\mathrm{BI}^{-/-}$mice exhibited similar atherogenic lipid levels and lipoprotein-cholesterol distribution, the latter developed 1.5 -fold more arterial lesions, supporting the proposal that expression of SR-BI in peripheral tissues may fulfill an atheroprotective function.

Generation of conditional SR-BI-deficient and SR-BI-/- mice. Conditional targeting of the mouse SR-BI gene was achieved by flanking exon 1 with Cre recombinase lox $\mathrm{P}$ sites following homologous recombination in 129/Sv ES cells (Figure 1A). The SR-BI floxed region includes $85 \mathrm{bp}$ of untranslated sequence, the entire coding region of the first exon, and approximately $3.5 \mathrm{~kb}$ of intron 1 . SR-BI exon 1 was previously deleted in mice and resulted in a null allele (10). Thus, Cre-mediated deletion of SR-BI floxed exon 1 was appropriate for achieving SR-BI inactivation. Several ES cell clones that were found to be correctly targeted by PCR and Southern blot analysis and that contained a floxed SR-BI allele (SR-BI $I^{\text {flox/+ }}$ ES clones) were transfected with a vector encoding for the Cre recombinase in order to generate ES clones with an SR-BI-null allele 

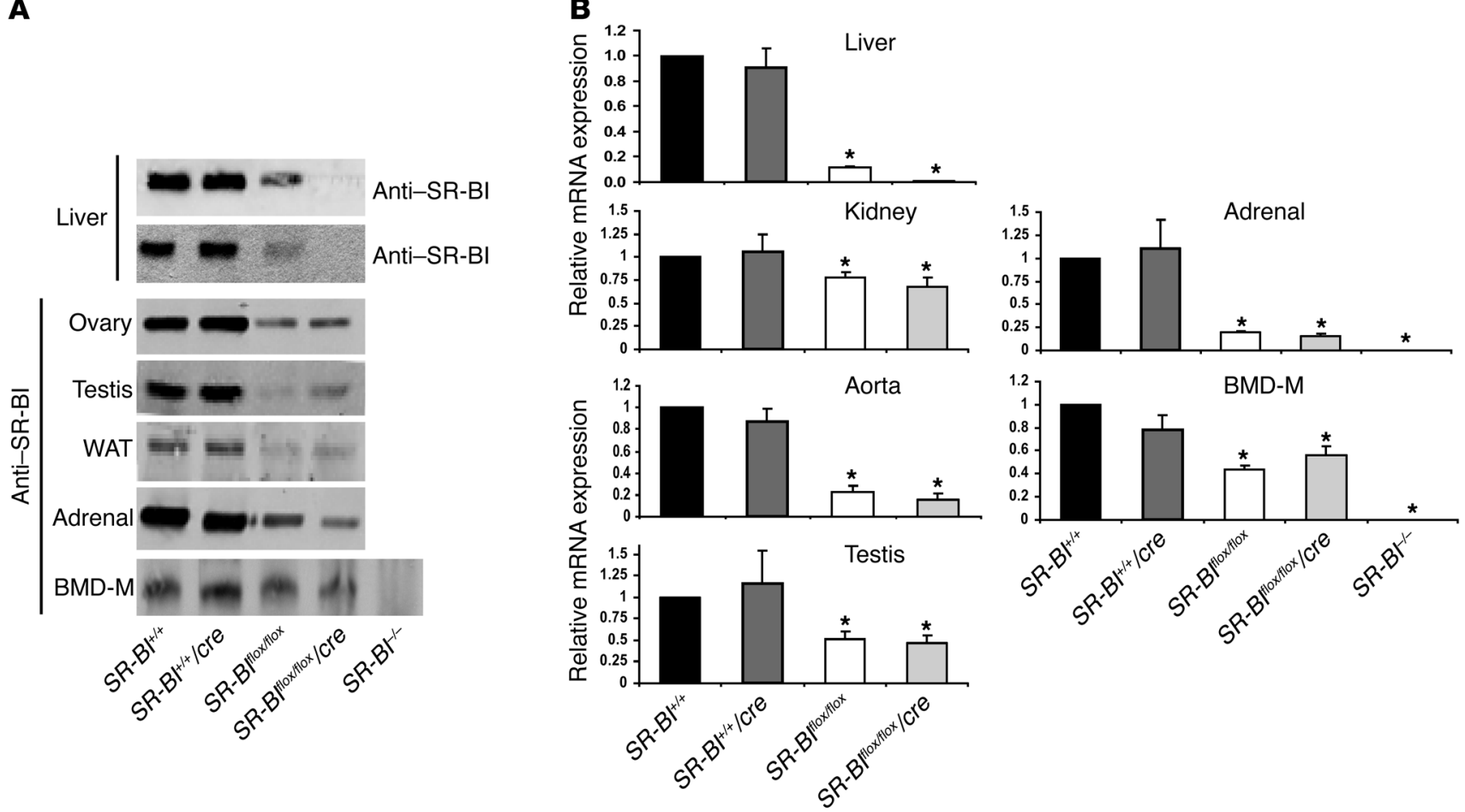

Figure 2

Levels of SR-BI expression. (A) For each SR-BI genotype, total protein extracts were prepared from the indicated tissues and loaded onto SDS-PAGE, followed by immunoblotting with an anti-SR-BI antibody (or with an anti-SR-BII antibody for liver extracts) directed against the C-terminal domain of the protein. WAT, white adipose tissue; BMD-M, BM-derived macrophages. (B) Relative SR-BI mRNA levels. Total RNAs extracted from tissues were pooled (4-8 mice per group), reverse-transcribed, and subjected to real-time PCR quantification. Values (mean \pm SD) represent the amount of SR-BI mRNA relative to that measured in control mice, which was set arbitrarily to 1. Data for BM-derived macrophages were generated from 1 representative RNA preparation for each SR-BI genotype. ${ }^{\star} P<0.05$ compared with controls.

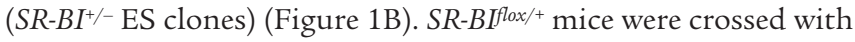
Alb-Cre transgenic mice (29), which express the Cre transgene under the albumin promoter for liver-specific expression, in order to generate $S R-B I^{f l o x /+} /$ Cre progeny. These animals were then backcrossed for 6 generations against C57BL/6J mice and subsequently interbred to yield $S R-B I^{+/+}, S R-B I^{+/+} / C r e, S R-B I^{\text {flox/flox }}$, and $S R-B I^{\text {flox/flox } / ~}$ Cre mice. The Alb-Cre transgene was systematically maintained in a hemizygous state throughout the study. $S R-B I^{+/-}$mice used in this study were also backcrossed on the C57BL/6J genetic background for 6 generations.

Alb-Cre transgenic mice have been previously described to provide complete and specific Cre-mediated recombination of floxed genes in adult hepatocytes $(29,30)$. PCR analysis of tail genomic DNA of $S R-B I^{\text {flox/flox } / C r e ~ m i c e ~ s h o w e d ~ t h e ~ p r e s e n c e ~ o f ~ o n l y ~ f l o x e d ~}$ alleles, whereas both floxed (to a minor degree) and knockout alleles were detected by PCR amplification of liver genomic DNA preparation (Figure 1C). We attribute the presence of the PCR product corresponding to the floxed allele in PCR amplifications from liver DNA of $S R-B I^{\text {flox/flox } / C r e ~ m i c e ~ t o ~ g e n o m i c ~ D N A ~}$ from nonhepatic cells, including endothelial and Kupffer cells, that are naturally present in liver tissue. In $S R-B I^{-/-}$mice, only the $\mathrm{KO}$ allele was amplified by PCR from both tail and liver tissues (Figure 1C). Western blot analysis of liver extracts of SR-BI $I^{\text {flox/flox } / ~}$ Cre mice showed essentially undetectable levels of SR-BI protein. In addition, the C-terminal variant of SR-BI, the SR-BII isoform, was not detectable in liver extracts (Figure 2A). qRT-PCR analysis dem- onstrated a decrease in SR-BI mRNA in the liver of $S R-B I^{\text {flox/flox}} /$ Cre mice to levels corresponding to $1 \%$ of those measured in $S R-B I^{+/+}$ mice (Figure 2B). No significant SR-BI signal was revealed by immunoblotting or by qRT-PCR upon analysis of tissues prepared from $S R-B I^{-1-}$ mice (Figure 2). Immunoblotting also clearly demonstrated that SR-BI expression was decreased significantly in the

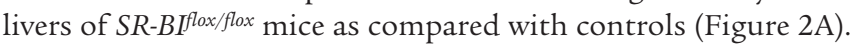
This decrement in SR-BI expression was confirmed by qRT-PCR analysis ( $-88 \%$ versus controls; Figure $2 \mathrm{~B})$. Analyses of other tissues confirmed that the expression of SR-BI was decreased in both

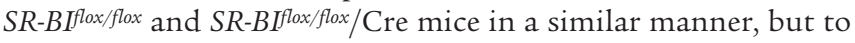
a different degree as a function of the tissue analyzed (Figure 2). These results therefore suggested that lox $\mathrm{P}$ targeting led to an attenuation of SR-BI mRNA and protein levels, thereby generating an hypomorphic SR-BI allele (hypomSR-BI allele). SR-BIflox/flox mice were consequently termed hypomSR-BI throughout this study. SR-BIflox/flox/Cre mice, which displayed a similar pattern of attenuated SR-BI expression in peripheral tissues compared with hypomSR-BI mice (Figure 2) but which were deficient for SR-BI in hepatocytes, were termed hypomSR-BI-KO ${ }^{\text {liver }}$ mice. Although we did not determine the origin of the reduced expression of the floxed SR-BI allele, the loxP site located in the $5^{\prime}$-UTR region of the SR-BI gene introduces a hairpin structure in the transcribed RNA that could explain attenuated downstream gene expression. However, we cannot exclude the possibility that expression of SR-BI may be influenced by the neomycin cassette. 
Table 1

Plasma lipid levels in mice fed a chow diet

\begin{tabular}{lcccccc} 
Genotype & Sex & $\boldsymbol{n}$ & $\mathbf{T C}(\mathbf{m g} / \mathbf{d l})$ & $\mathbf{F C}(\mathbf{m g} / \mathbf{d l})$ & $\mathbf{F C} / \mathbf{T C}(\%)$ & $\mathbf{T G}(\mathbf{m g} / \mathbf{d l})$ \\
Controls & $\mathrm{F}$ & 20 & $72 \pm 10$ & $17 \pm 4$ & $23 \pm 3$ & $74 \pm 24$ \\
& $\mathrm{M}$ & 16 & $90 \pm 12$ & $26 \pm 3$ & $29 \pm 2$ & $69 \pm 18$ \\
hypomSR-BI & $\mathrm{F}$ & 11 & $135 \pm 20^{\mathrm{A}}$ & $34 \pm 4^{\mathrm{A}}$ & $26 \pm 4^{\mathrm{B}}$ & $73 \pm 19$ \\
& $\mathrm{M}$ & 10 & $175 \pm 33^{\mathrm{A}}$ & $56 \pm 9^{\mathrm{A}}$ & $32 \pm 5$ & $85 \pm 35$ \\
hypomSR-BI-KOliver & $\mathrm{F}$ & 12 & $168 \pm 22^{\mathrm{A}}$ & $62 \pm 8^{\mathrm{A}}$ & $37 \pm 6^{\mathrm{A}}$ & $86 \pm 26$ \\
& $\mathrm{M}$ & 11 & $194 \pm 27^{\mathrm{A}}$ & $87 \pm 12^{\mathrm{A}}$ & $45 \pm 5^{\mathrm{A}}$ & $77 \pm 40$ \\
SR-Bl-- & $\mathrm{F}$ & 10 & $137 \pm 13^{\mathrm{A}, \mathrm{C}}$ & $53 \pm 8^{\mathrm{A}, \mathrm{D}}$ & $39 \pm 9^{\mathrm{A}, \mathrm{E}}$ & $72 \pm 26$ \\
& $\mathrm{M}$ & 8 & $156 \pm 5^{\mathrm{A}, \mathrm{E}}$ & $87 \pm 4^{\mathrm{A}, \mathrm{E}}$ & $55 \pm 3^{\mathrm{A}, \mathrm{C}}$ & $79 \pm 29^{\mathrm{E}}$ \\
\hline
\end{tabular}

Plasma TC, FC, and TG levels were measured in 2-month-old female (F) and male (M) mice fed a chow diet. Data represent mean \pm SD. ${ }^{A} P<0.001$ versus control mice. ${ }^{B} P<0.04$ versus control mice. ${ }^{C} P<0.002$ versus hypomSR-BI-KOliver mice. ${ }^{D} P<0.03$ versus hypom$\mathrm{SR}-\mathrm{BI}-\mathrm{KO}$ liver mice. ENot statistically different from values for hypomSR-BI-KOliver mice.

$(10,11)$. Thus, significantly reduced expression of SR-BI in hypomSR-BI mice, and in particular in the liver, resulted in a marked increase in plasma total cholesterol (TC) concentrations in both males and females (Table 1). Indeed, TC levels in these mice were increased to the same order of magnitude as those in $S R-B I^{-/-}$mice, the latter being almost 2 -fold higher compared with the levels in controls. This 2-fold increase in plasma TC levels was similar to that reported in an SR-BI-KO mouse line described earlier $(10,15)$. Plasma TC concentrations in hypomSR-BI-KO ${ }^{\text {liver }}$ mice tended to be higher. Interestingly, the 2-fold elevation of plasma TC levels in hypomSR-BI mice was associated with a corresponding 2 -fold increase in $\mathrm{FC}$, whereas plasma FC levels were increased more than 3-fold in both $S R-B I^{-/-}$and hypomSR-BI-KOliver mice (Table 1 ). As a consequence, both $\mathrm{SR}-\mathrm{BI}^{-/-}$and hypomSR-BI-KOliver

It has been previously reported that matings of heterozygous or homozygous SR-BI-KO males with SR-BI-KO female mice are nonproductive due to female sterility (16). Moreover, intercrosses of $\mathrm{SR}-\mathrm{BI}^{+/-}$mice on a mixed C57BL6/129 background result in the transmission of the SR-BI-KO allele in a non-Mendelian ratio, with a low frequency for the production of $S R-B I^{-1-}$ mice (10). Generation of homozygous SR-BI-KO mice on a pure C57BL/6 background has equally been reported to be difficult (31). We observed similar results with our SR-BI-KO mouse line, as female $S R-B I^{-/-}$ mice were sterile, and we could not generate SR-BI-KO mice in a C57BL/ 6 background following the mating of $S R-B I^{+/-}$female and male mice fed a normal chow diet. Fertility in SR-BI-KO females was restored and SR-BI-KO pups were obtained from heterozygous breeding pairs when animals were fed a chow diet supplemented with the lipid-lowering drug probucol $(0.5 \%)$, as previously reported $(21,31)$. The frequency of the genotypes derived from mating of $S R-B I^{f l o x /+}$ and $S R-B I^{f l o x /+} /$ Cre mice fed a normal chow diet was close to that expected for Mendelian inheritance $\left(S R-B I^{+/+}, 16 \%\right.$; $S R-B I^{+/+} /$Cre, 11\%; SR-BIflox/+, $24 \%$; SR-BI $I^{\text {flox/++}} /$ Cre, 30\%; SR-BI flox/flox [hypomSR-BI], 10\%; and SR-BIflox/flox/Cre [hypomSR-BI-KOliver], 9\%; $n=210$ ). Additionally, hypomSR-BI-KO ${ }^{\text {liver }}$ female mice produced normal progeny without diet supplementation with probucol. These observations indicate that extrahepatic expression of SR-BI, most likely in ovaries or in developing oocytes $(16,28,31)$, is required for female fertility in mice.

SR-BI dose-dependent effect on plasma cholesterol levels. Plasma lipids were analyzed in mice on a chow diet at 2 months of age. As reported previously, targeted inactivation ( $\left.S R-B I^{-/-}\right)$, hemizygous expression $\left(S R-B I^{+/-}\right)$, or attenuated expression of SR-BI in mice results in a gene dose-dependent increase in plasma levels of HDL-C

\section{Figure 3}

Impact of changes in SR-BI expression on plasma lipoprotein cholesterol profiles. (A) Fasting plasma samples (pooled plasma from 6-10 mice) from 2-month-old female mice fed a chow diet were fractionated by size-exclusion chromatography. TC was measured in each fraction to determine lipoprotein cholesterol profiles. Approximate elution volumes for particles in the size ranges of VLDL, LDL, and HDL are indicated. (B) Electrophoretic mobilities of plasma lipoproteins. Plasma samples $(10 \mu \mathrm{l})$ were subjected to agarose gel electrophoresis and lipoproteins revealed by Sudan black staining. $\alpha$, pre- $\beta$, and $\beta$ positions corresponding to migrations of HDL, VLDL, and LDL particles, respectively, are indicated. lines exhibited a high plasma FC/TC ratio; by contrast, this ratio was either moderately increased or similar in hypomSR-BI females and males, respectively, compared with controls (Table 1). These data clearly demonstrate that hepatic SR-BI deficiency results in a specific accumulation of FC in plasma. As shown in Figure 2, and as previously reported (10), changes in plasma cholesterol levels reflected a marked elevation of HDL-C in $S R-B I^{-/-}$mice with abnormally large HDL particles. A similar lipoprotein cholesterol profile was observed in hypomSR-BI-KO ${ }^{\text {liver }}$ mice, particularly with respect to the cholesterol content of large HDL particles. apoE was more abundant in these particles as compared with HDL of normal size (data not shown), as previously reported for large HDL present in $S R-B I^{-/-}$mice (10). Analysis of the cholesterol elution profile of plasma from hypomSR-BI mice also revealed elevation in HDL-C levels, as expected, although large HDL particles appeared consistently more heterogeneous in size as compared with those apparent in both $S R-\mathrm{BI}^{-1-}$ and hypomSR-BI-KO liver profiles (Figure 3A). Lipoprotein cholesterol distribution was similar in male and

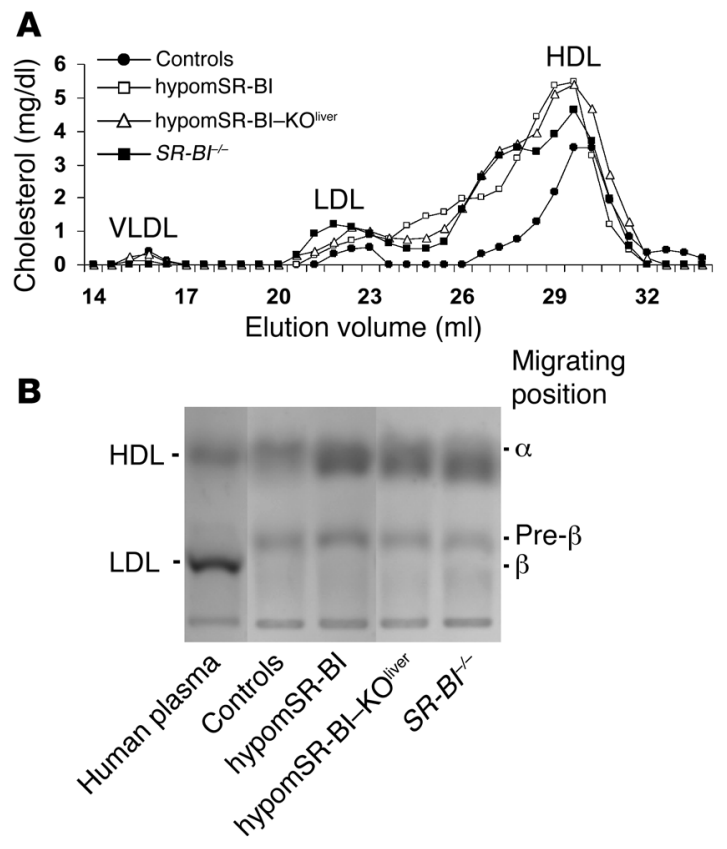


Table 2

Plasma lipid levels in female mice fed the atherogenic HFC diet

$\begin{array}{lccccc}\text { Genotype } & \boldsymbol{n} & \text { TC (mg/dl) } & \text { FC (mg/dl) } & \text { FC/TC (\%) } & \text { TG (mg/dl) } \\ \text { Controls } & 20 & 160 \pm 38 & 51 \pm 15 & 32 \pm 5 & 27 \pm 6 \\ \text { hypomSR-BI } & 11 & 374 \pm 97^{\mathrm{A}} & 183 \pm 61^{\mathrm{A}} & 48 \pm 8^{\mathrm{A}} & 33 \pm 10 \\ \text { hypomSR-BI-KOliver } & 12 & 1,119 \pm 252^{\mathrm{A}} & 839 \pm 224^{\mathrm{A}} & 75 \pm 10^{\mathrm{A}} & 60 \pm 21^{\mathrm{A}} \\ \text { SR-B } \text { H }^{--} & 10 & 969 \pm 151^{\mathrm{A}, \mathrm{B}} & 707 \pm 128^{\mathrm{A}, \mathrm{B}} & 73 \pm 8^{\mathrm{A}, \mathrm{B}} & 48 \pm 8^{\mathrm{A}, \mathrm{B}}\end{array}$

Plasma TC, FC, and TG levels were measured in female mice after feeding an HFC diet for 11 weeks. Data represent mean $\pm S D$. ${ }^{A} P<0.001$ versus control mice. ${ }^{B}$ Not statistically different from hypomSR-BI-KOliver mice.

such as SREBP1, SREBP2, LXR, FXR, and LRH-1 (data not shown). However, we confirmed an increase in hepatic mRNA levels for the ABCG subfamily member ABCG1 in SR-BI-/- mice (15) (1.9-fold increase versus controls; $P<0.0001)$. An increase of similar magnitude was observed in the livers of hypomSR-BI-KOliver mice (1.7-fold versus controls; $P<0.001)$ but not in that of hypomSR-BI animals. Interestingly, both $S R-B I^{-/-}$and hypomSR-BI$\mathrm{KO}^{\text {liver }}$ mice exhibited a 1.5 -fold elevation $(P<0.001$ vs controls) in hepatic mRNA levels for the macrophage marker F4/80, while no change could be detected in liver RNA prepared from hypomSR-BI

female groups. Interestingly, analysis of electrophoretic mobilities revealed that larger HDL particles in hypomSR-BI, hypomSR-BI$\mathrm{KO}^{\text {liver }}$, and $\mathrm{SR}-\mathrm{BI}^{-/-}$mice displayed slightly lower electronegativity compared with the normal-sized HDL present in control mice, potentially reflecting differences in apolipoprotein content (Figure 3B). Finally, no differences in plasma triglyceride (TG) levels were observed among SR-BI genotypes (Table 1).

SR-BI dose-dependent effect on cholesterol homeostasis in liver. As reported previously (15), the hepatic content of FC and esterified cholesterol in $S R-\mathrm{BI}^{-/-}$mice did not differ from those in controls. Similar results were also observed for both hypomSR-BI and hypomSR-BI-KO ${ }^{\text {liver }}$ mice. In addition, no significant difference was observed among SR-BI genotypes in hepatic mRNA levels of LDL-r, ABCA1, apoB-100, SR-BI-interacting scaffold protein PDZK1, and transcriptional regulators of hepatic lipid metabolism, mice. Taken together, these observations suggest that elevated ABCG1 expression in livers of $S R-B I^{-/-}$and hypomSR-BI-KOliver mice may result from increased numbers and/or activation of resident hepatic macrophages.

SR-BI dose-dependent effect on the development of atherosclerosis. To evaluate the impact of general knockdown expression of SR-BI on atherosclerosis susceptibility and, in addition, to assess the potential supplementary effect triggered by complete deletion of hepatic SR-BI in this context, the various mouse lines were fed a high-fat, high-cholesterol, and cholic acid-containing (HFC) diet for 11 weeks. This diet resulted in an elevation in plasma TC (>2fold) and a decrease in TG levels (>2.5-fold) of similar magnitude in hypomSR-BI and control mice (Tables 1 and 2). Plasma TC levels were significantly raised in both hypomSR-BI-KO ${ }^{\text {liver }}$ and $S R-B I^{-/-}$mice, mostly as a consequence of elevated concentrations
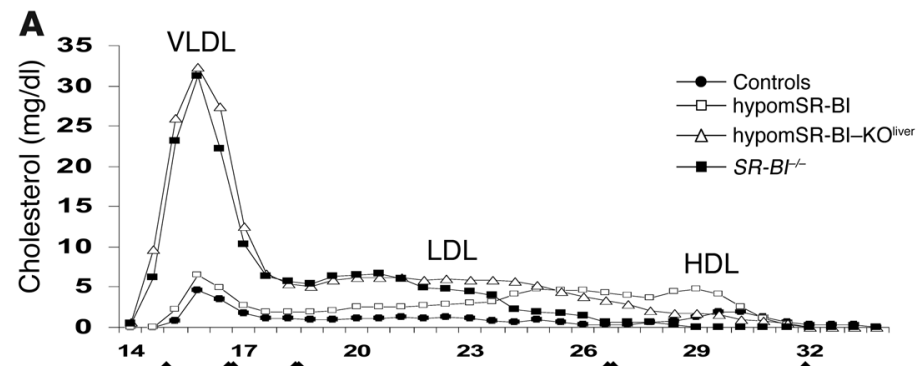

$\rightarrow-S R-B t^{-}$

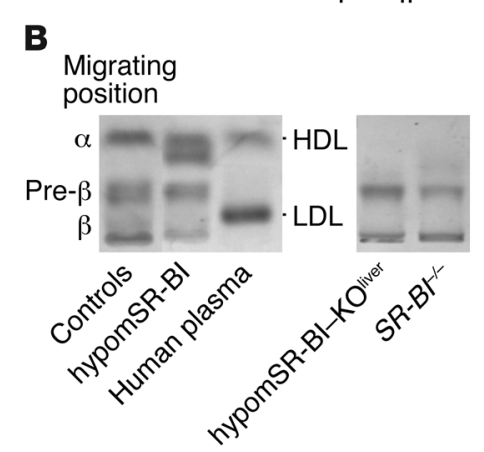

Elution volume $(\mathrm{ml})$

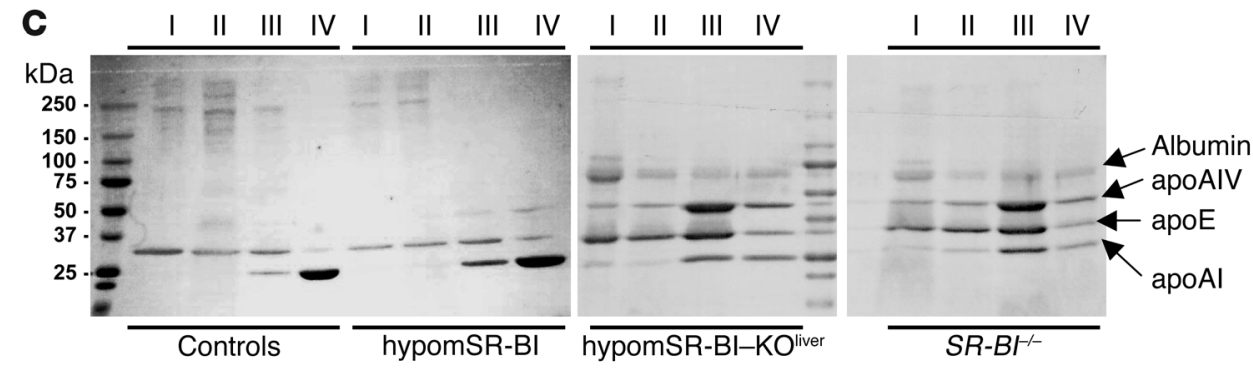

Figure 4

Analysis of the distribution of plasma lipoprotein cholesterol in female mice fed an atherogenic diet. (A) Lipoprotein cholesterol profiles. Fasting plasma samples were prepared from female mice fed an atherogenic (HFC) diet for 11 weeks. For each genotype, plasma samples from individual mice were pooled and loaded on Superose 6 columns, and cholesterol was measured in collected fractions. (B) Electrophoretic mobilities of plasma lipoproteins. Plasma samples $(10 \mu \mathrm{l})$ were subjected to agarose gel electrophoresis and lipoproteins revealed by Sudan black staining. (C) Apolipoprotein composition of lipoprotein fractions. Lipoproteins were first isolated from pooled plasmas $(n=5-10)$ by ultracentrifugation at a density of $1.21 \mathrm{~g} / \mathrm{ml}$ and then fractionated by size-exclusion chromatography. Collected fractions were pooled as indicated in A (I, II, III, and IV), concentrated, and subjected (20-30 $\mu$ g protein loaded per lane) to SDS-PAGE on gradient gels. Proteins were visualized by Coomassie blue staining. Bands corresponding to apoAI, apoE, and apoAIV, as confirmed by immunoblotting (data not shown), are indicated. 
A

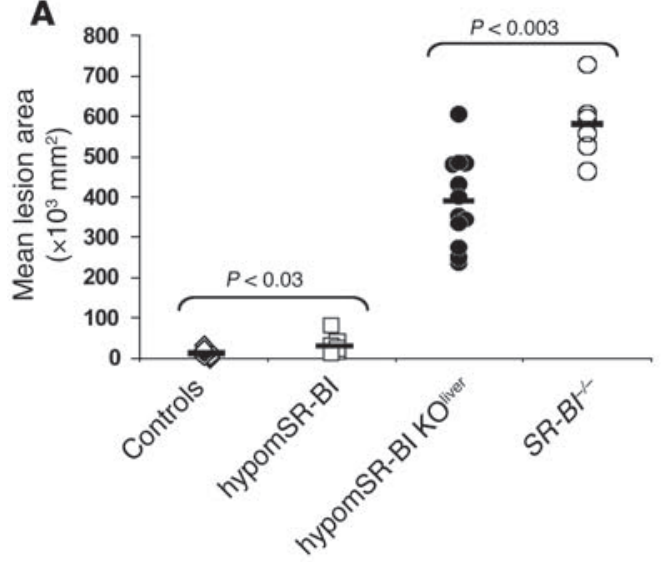

C
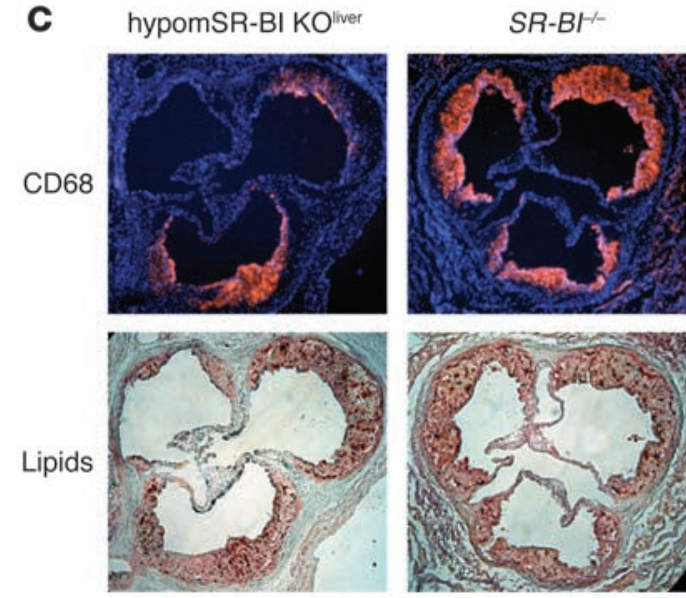

B

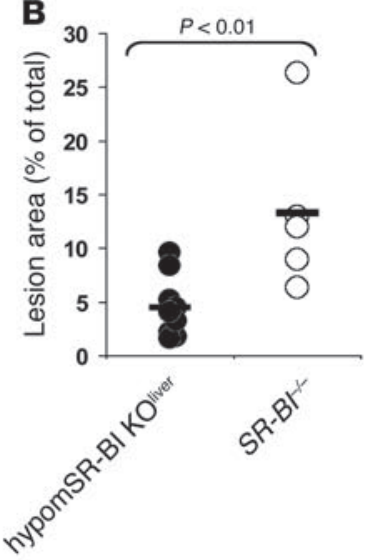

D

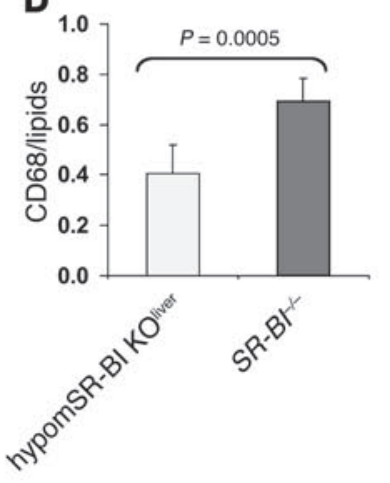

$\mathbf{E}$

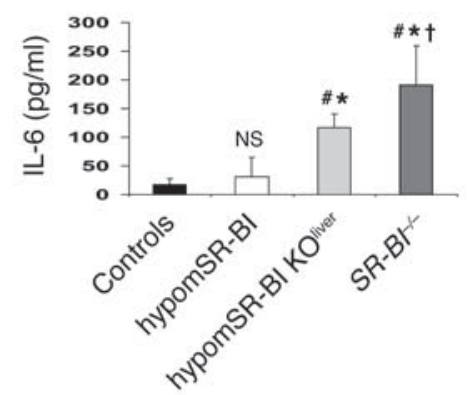

\section{Figure 5}

Quantification of atherosclerosis and inflammation in mice fed the atherogenic HFC diet. (A) Degree of atherosclerosis in the aortic root area. Lipid deposits were visualized by oil red $\mathrm{O}$ staining of aortic root sections. Each symbol represents the mean lesion area in a single mouse. The horizontal bar indicates the mean value for the group. Statistically significant differences between groups are indicated. (B) Quantification of lipid lesions in the aortic arch and thoracic aorta of hypomSR-BI-KOliver and SR-BI-l- mice. Each symbol represents the percentage of the aorta stained for lipid with Sudan IV in a single mouse. The horizontal bar indicates the mean value for each group. Statistically significant differences between the 2 groups are indicated. No lipid staining could be detected in controls and in hypomSR-BI mice. (C) Increased monocyte/macrophage accumulation in atherosclerotic lesions of $S R-\mathrm{Bl}^{--}$mice as compared with hypomSR-BI-KOliver mice. Aortic atherosclerotic lesions were immunostained for monocyte/macrophage CD68 antigen (upper panels; nuclei are visualized in blue with DAPI staining) and subsequently processed for lipid staining with oil red $\mathrm{O}$ (lower panels). (D) The surface ratio of CD68+ staining to oil red O-positive staining was calculated for both groups. Values represent the mean \pm SD of 6 size-matched lesions analyzed per group. (E) IL-6 plasma levels in HFC diet-fed mice. ${ } P<0.03$ compared with controls; ${ }^{\star} P<0.05$ compared with hypomSR-BI mice; ${ }^{\dagger} P<0.05$ compared with hypomSR-BI-KOliver mice. NS, not statistically different compared with values for controls.

of plasma FC. Indeed, the plasma FC/TC ratio was increased 2-fold as compared with the chow-fed condition in both lines and was 1.5 and 2.3-fold greater relative to that observed in hypomSR-BI and control female mice fed the atherogenic diet, respectively. Plasma TG levels were equally elevated in both HFC diet-fed hypomSR$\mathrm{BI}-\mathrm{KO}^{\text {liver }}$ and $\mathrm{SR}-\mathrm{BI}^{-/-}$mice as compared with controls. Importantly, plasma TC, FC, and TG concentrations, as well as the FC/TC ratio, were not statistically different between hypomSR-BI-KO ${ }^{\text {liver }}$ and $S R-B I^{-/-}$mice after the atherogenic diet (Table 2). Analysis of plasma lipoprotein cholesterol profiles revealed that the amount of cholesterol associated with VLDL-sized particles was similarly increased in both control and hypomSR-BI mice when fed the HFC diet. However, higher levels of plasma TC in hypomSR-BI mice as compared with controls was primarily due to greater amounts of cholesterol associated with both large and normal-sized HDL
(Figure 4A). As seen under normal chow diet feeding (Figure 3B), large HDL particles present in HFC diet-fed hypomSR-BI mice exhibited a lower electronegativity on agarose gels as compared with normal HDL (Figure 4B). Lipoprotein cholesterol profiles of hypomSR-BI-KOliver and $S R-B I^{-/-}$mice fed the HFC diet were similar, cholesterol being mainly associated with VLDL-sized particles. Analysis of these particles from both lines revealed that they exhibited similar characteristics, as they migrated to the pre- $\beta$ position in agarose gels (Figure $4 \mathrm{~B}$ ) and were rich in apoE and, to a lesser extent, in apoAIV (Figure 4C). The FC/TC ratios in these particles were also similar (73\% and 78\% for hypomSR-BI-KOliver and $S R-B I^{-1-}$ mice, respectively) and reflected those measured in the plasma of both lines (Table 2). By comparison, FC/TC ratios in isolated VLDL-sized particles from hypomSR-BI and control mice were $34 \%$ and $21 \%$, respectively. To further characterize the 
apoE-rich VLDL-sized particles migrating to the pre- $\beta$ position present in the plasma of both HFC diet-fed hypomSR-BI-KOliver and $S R-B I^{-1-}$ mice, we compared their capacity to induce cholesterol loading in the murine macrophage RAW cell line. The increase in cellular esterified cholesterol and FC content following incubation of RAW cells with these particles was similar for both mouse lines (see Supplemental Figure 1; supplemental material available online with this article; doi:10.1172/JCI26893DS1). Finally, analysis of the lipoprotein cholesterol profiles of hypomSR-BI-KOliver and $S R-B I^{-/-}$mice fed the HFC diet also revealed that HDL-C levels were markedly reduced in both lines as compared with that present in hypomSR-BI mice; such reduction occurred primarily in normal-sized HDL (Figure 4A). This observation was confirmed by agarose gel analysis (Figure 4B). In addition, SDS-PAGE analysis of lipoproteins showed significantly increased amounts of both apoE and apoAIV in the protein moieties of HDL particles in both hypomSR-BI-KOliver and $S R-B I^{-/-}$mice (Figure 4C).

SR-BI knockdown expression in hypomSR-BI mice led to a 2.5 -fold $(P<0.03)$ increase in mean atherosclerotic lesion area as compared with that in wild-type mice $\left(12 \times 10^{3} \pm 10 \times 10^{3} \mu \mathrm{m}^{2}\right.$ and $30 \times 10^{3} \pm 22 \times 10^{3} \mu \mathrm{m}^{2}$, respectively; Figure $5 \mathrm{~A}$ ). A further 13 -fold increase in the size of lipid lesions $\left(389 \times 10^{3} \pm 112 \times 10^{3} \mu \mathrm{m}^{2}\right)$ was observed in hypomSR-BI-KOliver mice, thus confirming the atheroprotective role of hepatic SR-BI expression, as previously reported $(6,17)$, but equally emphasizing its importance. Finally, analysis of $S R-B I^{-1-}$ mice revealed increased susceptibility to atherosclerotic lesion development as compared with hypomSR-BI-KOliver animals, with a mean lesion area of $579 \pm 89 \times 10^{3} \mu \mathrm{m}^{2}$ (1.5-fold increase compared with hypomSR-BI-KOliver mice; $P<0.003$; Figure $5 \mathrm{~A})$. These findings were confirmed by lesion size quantification with en face analysis of thoracic and abdominal aortas (Figure 5B). Lipid lesions covered an average of $4.5 \% \pm 2.8 \%$ and $13.3 \% \pm 7.7 \%$ of the total aortic surface analyzed in hypomSR-BI-KOliver and $S R-B I^{-1-}$ mice, respectively; this difference was statistically significant $(-66 \%$ in hypomSR-BI-KO ${ }^{\text {liver }}$ versus $S R-B I^{-/-}$mice; $P<0.01$ ).

To examine the cellular content of aortic root lesions, and in particular macrophage abundance, we stained selected sections adjacent to size-matched lesions of hypomSR-BI-KO $\mathrm{K}^{\text {liver }}$ and $S R-B I^{-/}$mice (as determined by oil red $\mathrm{O}$ staining) for $\mathrm{CD} 68$ antigen and then further processed them for oil red O lipid staining (Figure 5C). Surprisingly, the average surface ratio of $\mathrm{CD} 8^{+}$staining to lipid-positive staining was statistically significantly lower $(-40 \%)$ in hypomSR-BI-KOliver mice than in $S R-B I^{-/-}$mice $(0.4 \pm 0.1$ vs. $0.7 \pm 0.1$, respectively; $P=0.0005)$ (Figure 5D). These data suggested an increased accumulation of macrophages, reflecting increased vascular inflammation in lesions of $S R-B I^{-/-}$mice as compared with hypomSR-BI-KOliver mice. Concomitantly, plasma concentrations of the proinflammatory cytokine IL-6 were elevated to a greater degree in $S R-B I^{-/}$mice than in hypomSR-BI-KOliver mice fed the HFC diet (Figure 5E). Indeed, IL-6 plasma levels were increased 10.8- and 6.6-fold in $S R-B I^{-/-}$ and hypomSR-BI-KO ${ }^{\text {liver }}$ mice, respectively, as compared with control mice. HypomSR-BI mice also displayed a trend toward higher IL-6 levels (1.8-fold increase versus controls); however, such concentrations were not statistically different from those in controls.

\section{Discussion}

Our present investigations have revealed that whereas marked reduction in the expression of SR-BI in hypomSR-BI mice was associated with increased diet-induced atherosclerosis, complete hepatic SR-BI deficiency in hypomSR-BI-KOliver mice strongly impacted lipoprotein cholesterol distribution and massively accelerated atherosclerosis. Furthermore, comparison of hypomSR-BI-KOliver mice and $S R-B I^{-/-}$mice demonstrated that, in a similar atherogenic lipoprotein environment, residual peripheral SR-BI expression contributed to significant atheroprotection in hypomSR-BI-KO ${ }^{\text {liver }}$ mice. These data suggest that whereas the major atheroprotective role of hepatic SR-BI is tightly associated with its impact on plasma lipoprotein cholesterol levels, peripheral SR-BI expression may protect against atherosclerosis through mechanisms that are independent of changes in plasma lipid parameters.

Diet-induced atherogenesis in $S R-B I^{+/-}$mice (15) or mice with attenuated expression of SR-BI (32) revealed either no difference or a 2- to 3-fold increase in atherosclerosis development as compared with their respective SR-BI-expressing controls. In contrast, complete deficiency of SR-BI in mice was consistently associated with a major increase in atherosclerosis development $(15,21)$. This observation is consistent with the modest ( 2.5 -fold) and significant increases (48-fold) in atherosclerosis that we observed in hypomSR-BI and $S R-B I^{-1-}$ mice, respectively, as compared with controls. Residual SR-BI expression in mice may therefore ensure partial to complete atheroprotection. In contrast, the marked increase in atherosclerotic lesion size in hypomSR-BI-KO ${ }^{\text {liver }}$ mice, as compared with hypomSR-BI and control mice, further confirms the critical role of hepatic SR-BI in atherogenesis $(6,17,18)$, albeit under conditions of reduced expression of SR-BI in peripheral tissues, as in this study. Comparison of plasma lipoprotein cholesterol profiles indicates that such protection probably involves the contribution of hepatic SR-BI to the prevention of the accumulation of atherogenic plasma lipoproteins of VLDL size rich in FC. The specific increase in plasma FC/TC ratio observed in hypomSR-BI-KOliver and $S R-B I^{-1-}$ mice fed a chow diet suggests that hepatic SR-BI is a key determinant in maintaining plasma cholesterol homeostasis, most likely through its contribution to the delivery of HDL-CE $(33,34)$ and/or of HDL-FC $(8)$ to the liver. Expression of SR-BI, albeit at low levels, in livers of hypomSR-BI mice may have ensured such activities. Interestingly, PDZK1-deficient mice, which display a profound reduction in hepatic SR-BI protein expression ( $95 \%)$, possess elevated HDL-C plasma concentrations but do not exhibit high plasma FC/TC ratios (28). Residual expression of SR-BI and/ or expression of the SR-BII isoform in the livers of PDZK1-KO mice may explain phenotypic similarities of the PDZK1-deficient mice to our hypomSR-BI mouse line. Accumulation of VLDL-sized lipoproteins in HFC diet-fed hypomSR-BI-KO ${ }^{\text {liver }}$ and $S R-B I^{-/-}$mice may also result from other activities attributed to SR-BI. Indeed, in vivo studies implicate SR-BI in the removal of apoB-containing lipoproteins from the circulation $(7,35)$ and in postprandial chylomicron metabolism (36). Interestingly, however, we observed that these VLDL-sized lipoproteins contained primarily apoE rather than apoB100/B48. Whether such factors may contribute to the atherogenic plasma lipoprotein profile present in HFC diet-fed hypomSR-BI-KOliver and $S R-B I^{-/-}$mice has yet to be determined.

Strikingly, hypomSR-BI-KOliver mice displayed less extensive atherosclerosis than $S R-\mathrm{BI}^{-/-}$mice, despite the fact that the 2 mouse models exhibited similar plasma lipid levels, lipoprotein cholesterol distribution, and apolipoprotein composition. Moreover, VLDL-sized lipoproteins isolated from these models induced cholesterol loading of macrophage RAW cells to a similar degree. Thus, differences in the extent of atherosclerosis between the models appeared to reflect a plasma lipoprotein/cholesterol-independent mechanism. Reduced CD68 immunostaining in the plaques 
of hypomSR-BI-KO ${ }^{\text {liver }}$ mice may indicate attenuated vascular inflammation in this model, suggesting that extrahepatic SR-BI may exert antioxidant or/and antiinflammatory activities that attenuate atherosclerosis progression. Such activities may depend on the contribution of SR-BI to the uptake or metabolism of specific components transported by lipoproteins, such as the cellular antioxidant $\alpha$-tocopherol $(26,27)$ or the proinflammatory protein serum amyloid A (37), both of which may actively participate in atherogenesis $(38,39)$. Alternatively, in vivo evidence for a role of macrophage SR-BI expression in atherosclerosis has also been suggested by BM transplantation studies in mice (21-23). However, SR-BI in BM-derived cells could exert pro- or antiatherogenic activities depending on the specific stage of atherosclerosis development (23). Although these studies are not fully conclusive, as cell types other than macrophages may be derived from BM cells in vascular lesions (e.g., smooth muscle cells and endothelial cells; ref. 40), in vitro studies suggest that macrophage SR-BI could participate in atherogenesis via a spectrum of activities. Thus, SR-BI could be implicated in the clearance of apoptotic cells (25) or of atherogenic oxidized lipoproteins (41), activities that may contribute to prevention of the accumulation of inflammatory material in the arterial wall. SR-BI may also stimulate macrophage cholesterol efflux (23, 42 ) and thereby prevent foam cell formation. In this regard, it is noteworthy that production of the inflammatory cytokine IL- 6 is induced in macrophages as a consequence of cellular FC loading (43). Although plasma FC levels were dramatically and similarly increased in both HFC diet-fed hypomSR-BI-KOliver and $S R-B I^{-/}$ mice, IL-6 plasma levels were elevated to a greater extent in the latter. Determining whether complete absence of SR-BI in macrophages of $S R-B I^{-/-}$mice resulted in perturbation of cholesterol flux in lesion macrophages, which in turn contributed to higher IL-6 levels in these mice as compared with those measured in hypomSR-BI$\mathrm{KO}^{\text {liver }}$ mice, will be an important goal for future investigation.

In conclusion, this study not only further documents the involvement of hepatic and extrahepatic SR-BI expression in vivo in cholesterol homeostasis, but also provides new insight into the relationship between SR-BI and atherosclerosis susceptibility. Further experimentation using the SR-BI conditional-KO mouse model (hypomSR-BI mice) to define the relative contribution of SR-BI expressed by arterial wall cells, including endothelial cells and macrophages, to atherosclerotic lesion development may, however, be limited due to reduced expression of SR-BI in all tissues in this model. However, the significant difference between hypomSR-BI-KO ${ }^{\text {liver }}$ and $S R-B I^{-/-}$mice in atherosclerosis development at similar plasma lipid levels suggests that such mouse models may nonetheless represent a valuable tool with which to evaluate potential tissue-specific effects of SR-BI.

\section{Methods}

Generation of SR-BI conditional KO and SR-BI-/- mouse models. The left homology arm of the targeting vector was generated by PCR amplification using SRBI.f1 5'-TGAAGGTGGTCTTCAAGAGCAGTCCT-3' and SRBI. r1 5'-ACCTACACGGGGATCACGTTC-3' located $1.2 \mathrm{~kb}$ upstream and $3.5 \mathrm{~kb}$ downstream of SR-BI exon 1, respectively (Figure 1). A loxP sequence, 5'-GCAACTTCGTATAGCATACATTATACGAAGTTAT-3' (the outer 2 underlined bases were modified from the original loxP sequence in order to destabilize the lox $\mathrm{P}$ hairpin structure), was then introduced at position $-85 \mathrm{bp}$ (relative to the initiation translation codon). The left arm cassette containing the loxP site was then cloned into the PPN2T vector to yield the pPN2T-Larm plasmid. The right homology arm was PCR amplified using an approximately 8-kb SR-BI/BamHI fragment cloned in pBluescript as template and the following primer pair: SRBI/XhoI.loxP.f2 primer 5'-GCCGctcgagATAACTTCGTATAGCATACATTATACGAAGTTATGACAGTTTTCAGAGCTCAGGG-3' (complementary sequence adjacent to the SRBI.r1 primer sequence in intron 1), containing an introduced $5^{\prime}$ restriction site for XhoI (lowercase letters) and a $5^{\prime}$-loxP sequence (underlined), and M13 reverse primer 5'-CAGGAAACAGCTATGACC- $3^{\prime}$. The resulting PCR product was then subcloned into the PPN2T-Larm plasmid to yield the final vector $\mathrm{PPN} 2 \mathrm{~T}$-floxSRBI.

Mouse ES cells (129/SvJ; GoGermline; Genome Systems Inc.) were electroporated with 20-40 $\mu \mathrm{g}$ of PPN2T-floxSRBI construct and plated onto neomycin-resistant mouse embryonic fibroblast feeder layers. Selective medium was applied 2 days later. Individual clones were isolated after 10-12 days, expanded, and screened by Southern blot analysis of XbaI-digested DNA using probe A (Figure 1). Several targeted clones containing an SR-BI allele with a floxed exon $1\left(S R-B I^{f o x /+} \mathrm{ES}\right)$ were further transfected with pBS185 Cre plasmid (Invitrogen) modified to contain the hygromycin B resistance gene and subsequently selected in hygromycin B-containing medium. Cre-mediated deletion of exon 1 of the floxed SR-BI allele in hygromycin B-resistant ES clones ( $S R-B I^{+/-}$ES) was confirmed by PCR and Southern blot analysis. $S R-B I^{f o x /+}$ ES and derived $S R-B I^{+/-}$ES clones were injected into C57BL/6 blastocysts, and chimeric offspring were bred to $\mathrm{C} 57 \mathrm{BL} / 6$ mice to test for germline transmission of the floxed SR-BI allele or the exon 1-deleted SR-BI allele, respectively. $S R-B I^{f l o x /+}$ and $S R-B I^{+/-}$mouse lines selected for the study were derived from the same ES clone (i.e., one $S R-B I^{f l o x /+}$ ES clone and a derived $S R-B I^{+/-}$ES clone). Alb-Cre transgenic mice (29) were bred with mice carrying the SR-BI floxed allele to obtain animals with liver-specific deficiency of the SR-BI gene. $S R-B I^{-/-}$mice were generated by breeding heterozygous or homozygous animals fed a chow diet containing $0.5 \%$ probucol (Sigma-Aldrich). See Supplemental Methods for more detailed information on generation of the mouse models. The animals were housed in a conventional animal facility on a 6 am to $6 \mathrm{pm}$ dark/light cycle. They were weaned at 21 days and fed ad libitum a normal mouse chow diet (RM1; Dietex France). For the high-fat diet experiment, 2-month-old female mice were switched to a diet consisting of $1.25 \%$ cholesterol, $0.5 \%$ cholic acid, and $20 \%$ fat for 11 weeks. All animal procedures were performed at the Central Animal Facility of the Medical Faculty of La Pitié Hospital with approval from the Direction Départementale des Services Vétérinaires, Paris, France, under strict compliance with European Community Regulations.

Plasma and tissue analyses. Blood samples were collected in Microvette tubes (Sarstedt) before and after the high-fat diet following an overnight fast by retro-orbital bleeding using heparinized microhematocrit tubes. Plasma samples were stored frozen at $-80^{\circ} \mathrm{C}$. TC (Roche Diagnostics), FC (Wako), and TG (bioMérieux) concentrations were measured by enzymatic colorimetric assays. IL-6 plasma concentrations were measured with the ProteoPlex mouse cytokine kit (Novagen; EMD Biosciences). Plasma lipoproteins were fractionated by gel filtration on 2 Superose 6 (Amersham Biosciences) columns connected in series using a BioLogic DuoFlow Chromatography System (Bio-Rad). For apolipoprotein analysis by SDS-PAGE, plasmas were first ultracentrifuged $\left(100,000 \mathrm{~g}\right.$ for 5.5 hours at $15^{\circ} \mathrm{C}$ in a fixed-angle rotor in a TL100 ultracentrifuge [Beckman Coulter]) at a density of $1.21 \mathrm{~g} / \mathrm{ml}$. Lipoproteins in the supernatant were then fractionated by gel filtration on Superose 6 columns and collected into 4 fractions (I-IV). These fractions were concentrated on a Microcon YM-30 centrifugal filter unit (Millipore) and samples electrophoresed on 4\%-18\% SDS-polyacrylamide gels under reducing conditions. To determine cholesterol content in liver, total lipids were extracted from frozen livers $(\sim 30 \mathrm{mg})$ using the Folch extraction procedure (44). Cholesterol concentration was determined with a commercial enzymatic assay (Roche Diagnostics). Electrophoretic mobility of mouse lipoproteins was assessed on HYDRAGEL 7 LIPO + Lp(a) gels (Sebia). 
Immunoblot analysis. Mice were sacrificed by cervical dislocation. Organs were collected, rinsed in ice-cold PBS, and snap-frozen in liquid nitrogen. Frozen tissue specimens were disrupted and homogenized with a rotorstator homogenizer in ice-cold PBS containing $1 \%$ Triton and a protease inhibitor cocktail (Complete Mini protease inhibitor tablets; Roche Diagnostics). Samples were centrifuged at $10,000 \mathrm{~g}$ for 15 minutes at $4{ }^{\circ} \mathrm{C}$ and protein content determined in the supernatant with the bicinchoninic acid assay reagent (Pierce). Equal amounts of protein extracts were separated by electrophoresis on $8 \%$ SDS-polyacrylamide gels under reducing conditions and then blotted onto a Hybond-C super nitrocellulose membrane (Amersham Biosciences). SR-BI and SR-BII proteins were detected using a rabbit polyclonal antibody raised against a peptide corresponding to amino acids 496-509 of the murine SR-BI sequence (45) and a specific anti-SR-BII rabbit polyclonal antibody (NB 400-102; Novus Biologicals), respectively. Primary antibodies were detected using a peroxidase-conjugated anti-rabbit antibody and revealed by chemiluminescence (ECL Plus reagent; Amersham Biosciences).

Analysis of gene expression by qRT-PCR. RNAs were prepared from frozen tissue specimens using TRIzoL reagent (Invitrogen) and further purified with a NucleoSpin RNA II kit (Macherey-Nagel). cDNA preparation and quantitative PCR analysis were performed as previously described (45). The specific primers used are described in Supplemental Table 1.

Analysis of atherosclerotic plaques. Mice were sacrificed under isoflurane anesthesia and perfused with ice-cold PBS. Hearts were collected and fixed (Accustain 10\% formalin solution [Sigma-Aldrich] supplemented with $2 \mathrm{mM}$ EDTA and $20 \mu \mathrm{M}$ butylated hydroxytoluene at $\mathrm{pH}$ 7.4) for $30 \mathrm{~min}$ utes followed by overnight incubation in phosphate-buffered $20 \%$ sucrose solution at $4{ }^{\circ} \mathrm{C}$; hearts were subsequently embedded in Tissue-Tek OCT compound (Sakura). The entire aortas were fixed, cleaned of adipose and connective tissues, opened longitudinally, and pinned out on a wax surface. Atherosclerotic lesions were quantified both on pinned-open aortas by Sudan IV staining (thoracic and abdominal part of the aorta) and on serial cross-sections $(10 \mu \mathrm{m})$ through the aortic root by oil red $\mathrm{O}$ staining as previously described (46).

Immunohistochemistry. Ten-micrometer aortic root cryosections were air dried and fixed in formalin (10\%) for 30 minutes. Sections were then blocked for 60 minutes with 3\% BSA in PBS and then incubated with antiCD68 antibody or control antibodies (1:300 dilution in PBS with $20 \mathrm{mM}$ glycine; Serotec) overnight at $4{ }^{\circ} \mathrm{C}$. After washing, a goat anti-rat Ig biotinylated secondary antibody (1:300 dilution; BD Biosciences - Pharmingen) was added, followed by streptavidin-horseradish peroxidase. The signal was enhanced using the tyramide signal amplification (TSA) kit (NEL 702; PerkinElmer), and the sections were counterstained for nuclei with DAPI (Molecular Probes; Invitrogen).

Statistics. The statistical significance of the differences between groups was evaluated using 2-tailed Student's $t$ test for unpaired comparisons. $P<0.05$ was considered significant. Values are expressed as mean \pm SD.

\section{Acknowledgments}

This study was supported by the French National Institute for Health and Medical Research (INSERM) and by an International HDL Research Award from Pfizer to P. Lesnik and T. Huby. M.J. Chapman and P. Lesnik gratefully acknowledge the award of a "Contrat d'Interface" by the Assistance Publique - Hôpitaux de Paris/INSERM.

Received for publication September 16, 2005, and accepted in revised form July 18, 2006.

Address correspondence to: Thierry Huby, INSERM U551, Hôpital de la Pitié, 83 Bd de l'hôpital, 75651 Paris 13, France. Phone: 33-14217-78-60; Fax: 33-145-82-81-98; E-mail: huby@chups.jussieu.fr.
1. Marsche, G., et al. 2001. Identification of the human analog of SR-BI and LOX-1 as receptors for hypochlorite-modified high density lipoprotein on human umbilical venous endothelial cells. FASEBJ. 15:1095-1097.

2. Yuhanna, I.S., et al. 2001. High-density lipoprotein binding to scavenger receptor-BI activates endothelial nitric oxide synthase. Nat. Med. 7:853-857.

3. Yeh, Y.C., Hwang, G.Y., Liu, I.P., and Yang, V.C. 2002. Identification and expression of scavenger receptor SR-BI in endothelial cells and smooth muscle cells of rat aorta in vitro and in vivo. Atherosclerosis. 161:95-103.

4. Chinetti, G., et al. 2000. CLA-1/SR-BI is expressed in atherosclerotic lesion macrophages and regulated by activators of peroxisome proliferator-activated receptors. Circulation. 101:2411-2417.

5. Rhainds, D., and Brissette, L. 2004. The role of scavenger receptor class B type I (SR-BI) in lipid trafficking. Defining the rules for lipid traders. Int. J. Biochem. Cell Biol. 36:39-77.

6. Kozarsky, K.F., Donahee, M.H., Glick, J.M., Krieger, M., and Rader, D.J. 2000. Gene transfer and hepatic overexpression of the HDL receptor SR-BI reduces atherosclerosis in the cholesterol-fed LDL receptor-deficient mouse. Arterioscler. Thromb. Vasc. Biol. 20:721-727.

7. Ueda, Y., et al. 1999. Lower plasma levels and accelerated clearance of high density lipoprotein (HDL) and non-HDL cholesterol in scavenger receptor class B type I transgenic mice. J. Biol. Chem. 274:7165-7171.

8. Ji, Y., et al. 1999. Hepatic scavenger receptor BI promotes rapid clearance of high density lipoprotein free cholesterol and its transport into bile. J. Biol. Chem. 274:33398-33402.

9. Kozarsky, K.F., et al. 1997. Overexpression of the
HDL receptor SR-BI alters plasma HDL and bile cholesterol levels. Nature. 387:414-417.

10. Rigotti, A., et al. 1997. A targeted mutation in the murine gene encoding the high density lipoprotein (HDL) receptor scavenger receptor class B type I reveals its key role in HDL metabolism. Proc. Natl. Acad. Sci. U. S. A. 94:12610-12615.

11. Varban, M.L., et al. 1998. Targeted mutation reveals a central role for SR-BI in hepatic selective uptake of high density lipoprotein cholesterol. Proc. Natl. Acad. Sci. U. S. A. 95:4619-4624.

12. Osgood, D., et al. 2003. Genetic variation at the scavenger receptor class B type I gene locus determines plasma lipoprotein concentrations and particle size and interacts with type 2 diabetes: the Framingham study. J. Clin. Endocrinol. Metab. 88:2869-2879.

13. Hong, S.H., et al. 2002. Association between HaeIII polymorphism of scavenger receptor class B type I gene and plasma HDL-cholesterol concentration. Ann. Clin. Biochem. 39:478-481.

14. Acton, S., et al. 1999. Association of polymorphisms at the SR-BI gene locus with plasma lipid levels and body mass index in a white population. Arterioscler. Thromb. Vasc. Biol. 19:1734-1743.

15. Van Eck, M., et al. 2003. Differential effects of scavenger receptor BI deficiency on lipid metabolism in cells of the arterial wall and in the liver. J. Biol. Chem. 278:23699-23705.

16. Trigatti, B., et al. 1999. Influence of the high density lipoprotein receptor SR-BI on reproductive and cardiovascular pathophysiology. Proc. Natl. Acad. Sci.U.S. A. 96:9322-9327.

17. Ueda, Y., et al. 2000. Relationship between expression levels and atherogenesis in scavenger receptor class B, type I transgenics. J. Biol. Chem. 275:20368-20373.
18. Arai, T., Wang, N., Bezouevski, M., Welch, C., and Tall, A.R. 1999. Decreased atherosclerosis in heterozygous low density lipoprotein receptor-deficient mice expressing the scavenger receptor BI transgene. J. Biol. Chem. 274:2366-2371.

19. Zhang, Y., et al. 2005. Hepatic expression of scavenger receptor class B type I (SR-BI) is a positive regulator of macrophage reverse cholesterol transport in vivo. J. Clin. Invest. 115:2870-2874. doi:10.1172/ JCI25327.

20. Li, X.A., et al. 2002. High density lipoprotein binding to scavenger receptor, class B, type I activates endothelial nitric-oxide synthase in a ceramidedependent manner. J. Biol. Chem. 277:11058-11063.

21. Covey, S.D., Krieger, M., Wang, W., Penman, M., and Trigatti, B.L. 2003. Scavenger receptor class B type I-mediated protection against atherosclerosis in LDL receptor-negative mice involves its expression in bone marrow-derived cells. Arterioscler. Thromb. Vasc. Biol. 23:1589-1594.

22. Zhang, W., et al. 2003. Inactivation of macrophage scavenger receptor class B type I promotes atherosclerotic lesion development in apolipoprotein Edeficient mice. Circulation. 108:2258-2263.

23. Van Eck, M., Bos, I.S., Hildebrand, R.B., Van Rij, B.T., and Van Berkel, T.J. 2004. Dual role for scavenger receptor class B, type I on bone marrow-derived cells in atherosclerotic lesion development. Am.J. Pathol. 165:785-794.

24. Li, X.A., Guo, L., Dressman, J.L., Asmis, R., and Smart, E.J. 2005. A novel ligand-independent apoptotic pathway induced by scavenger receptor class $B$, type I and suppressed by endothelial nitric-oxide synthase and high density lipoprotein. J. Biol. Chem. 280:19087-19096.

25. Nakagawa, A., Shiratsuchi, A., Tsuda, K., and Nakanishi, Y. 2005. In vivo analysis of phagocytosis 
of apoptotic cells by testicular Sertoli cells. Mol. Reprod. Dev. 71:166-177.

26. Goti, D., et al. 2001. Scavenger receptor class B, type I is expressed in porcine brain capillary endothelial cells and contributes to selective uptake of HDLassociated vitamin E. J. Neurochem. 76:498-508.

27. Mardones, P., et al. 2002. Alpha-tocopherol metabolism is abnormal in scavenger receptor class B type I (SR-BI)-deficient mice. J. Nutr. 132:443-449.

28. Kocher, O., et al. 2003. Targeted disruption of the PDZK1 gene in mice causes tissue-specific depletion of the high density lipoprotein receptor scavenger receptor class B type I and altered lipoprotein metabolism. J. Biol. Chem. 278:52820-52825.

29. Postic, C., and Magnuson, M.A. 2000. DNA excision in liver by an albumin-Cre transgene occurs progressively with age. Genesis. 26:149-150.

30. Timmins, J.M., et al. 2005. Targeted inactivation of hepatic Abcal causes profound hypoalphalipoproteinemia and kidney hypercatabolism of apoA-I. J. Clin. Invest. 115:1333-1342. doi:10.1172/ JCI200523915

31. Miettinen, H.E., Rayburn, H., and Krieger, M. 2001. Abnormal lipoprotein metabolism and reversible female infertility in HDL receptor (SR-BI)-deficient mice. J. Clin. Invest. 108:1717-1722. doi:10.1172/ JCI200113288.

32. Huszar, D., et al. 2000. Increased LDL cholesterol and atherosclerosis in LDL receptor-deficient mice with attenuated expression of scavenger receptor B1.
Arterioscler. Thromb. Vasc. Biol. 20:1068-1073.

33. Brundert, M., et al. 2005. Scavenger receptor class B type I mediates the selective uptake of high-density lipoprotein-associated cholesteryl ester by the liver in mice. Arterioscler. Thromb. Vasc. Biol. 25:143-148.

34. Out, R., et al. 2004. Scavenger receptor class B type I is solely responsible for the selective uptake of cholesteryl esters from HDL by the liver and the adrenals in mice. J. Lipid Res. 45:2088-2095.

35. Wang, N., Arai, T., Ji, Y., Rinninger, F., and Tall, A.R. 1998. Liver-specific overexpression of scavenger receptor BI decreases levels of very low density lipoprotein ApoB, low density lipoprotein ApoB, and high density lipoprotein in transgenic mice. J. Biol. Chem. 273:32920-32926.

36. Out, R., et al. 2005. Adenovirus-mediated hepatic overexpression of scavenger receptor class B type I accelerates chylomicron metabolism in C57BL/6 mice. J. Lipid Res. 46:1172-1181.

37. Baranova, I.N., et al. 2005. Serum amyloid A binding to CLA-1 (CD36 and LIMPII analogous-1) mediates serum amyloid A protein-induced activation of ERK $1 / 2$ and p38 mitogen-activated protein kinases. J. Biol. Chem. 280:8031-8040.

38. O'Brien, K.D., et al. 2005. Serum amyloid A and lipoprotein retention in murine models of atherosclerosis. Arterioscler. Thromb. Vasc. Biol. 25:785-790.

39. Terasawa, Y., et al. 2000. Increased atherosclerosis in hyperlipidemic mice deficient in alpha-tocopherol transfer protein and vitamin E. Proc. Natl. Acad.
Sci. U. S. A. 97:13830-13834.

40. Sata, M., et al. 2002. Hematopoietic stem cells differentiate into vascular cells that participate in the pathogenesis of atherosclerosis. Nat. Med. 8:403-409.

41. Gillotte-Taylor, K., Boullier, A., Witztum, J.L., Steinberg, D., and Quehenberger, O. 2001. Scavenger receptor class B type I as a receptor for oxidized low density lipoprotein. J. Lipid Res. 42:1474-1482.

42. Ji, Y., et al. 1997. Scavenger receptor BI promotes high density lipoprotein-mediated cellular cholesterol efflux. J. Biol. Chem. 272:20982-20985.

43. Li, Y., et al. 2005. Free cholesterol-loaded macrophages are an abundant source of tumor necrosis factor-alpha and interleukin-6: model of NFkappaB- and map kinase-dependent inflammation in advanced atherosclerosis. J. Biol. Chem. 280:21763-21772.

44. Folch, J., Lees, M., and Sloane Stanley, G.H. 1957. A simple method for the isolation and purification of total lipides from animal tissues. J. Biol. Chem. 226:497-509.

45. Treguier, M., et al. 2004. Transcription factor sterol regulatory element binding protein 2 regulates scavenger receptor Cla-1 gene expression. Arterioscler. Thromb. Vasc. Biol. 24:2358-2364.

46. Lesnik, P., Haskell, C.A., and Charo, I.F. 2003. Decreased atherosclerosis in $\mathrm{CX}_{3} \mathrm{CR}^{-/-}$mice reveals a role for fractalkine in atherogenesis. J. Clin. Invest. 111:333-340. doi:10.1172/JCI200315555. 